\title{
Hydro-power plant equipped with Pelton turbines: basic experiments relating to the influence of backpressure on the design
}

\author{
A. Arch \& D. Mayr \\ Institute for Hydraulic Engineering and Water Resources Management, \\ Graz University of Technology, Austria
}

\begin{abstract}
Free-jet turbines working under backpressure conditions represent an economical alternative to conventional hydro-electric plant configurations. However, the air introduced into the tailwater generates an air/water mixture. Its reaction to a rising ambient pressure is at present being studied at the above Institute. This report deals with the effect an increase in ambient pressure has on the volume and consistency of the two-phase mixture and the rise velocity of air bubble swarms. In addition, a test set-up is described which was used to study the physical reaction of the water/air mixture to a change in ambient pressure conditions. The results and their effects on the configuration of free-jet turbines working under backpressure are discussed.
\end{abstract}

Keywords: air/water mixture, Pelton turbines, backpressure, bubble rise velocity.

\section{Introduction}

Recent developments have shown that multiple-jet Pelton turbines working under backpressure conditions in hydro-electric power plants may be an adequate choice in the case of (1) major tailwater-level oscillations, (2) full turbine operation between 0 and $100 \%$ being required without any major efficiency loss, and (3) the need to reduce the difference in turbine and pump levels to minimise construction cost.

Figure 1 is a section through the typical arrangement of a vertical-shaft backpressure Pelton turbine. As the jets emerging from the Pelton bucket hit the water surface in the tailrace channel, air is entrained and carried along into the 
tailwater, which causes an air/water mixture to form directly below the turbine, thus raising the level of the 2-phase mixture. Over the free-flow section which follows, the undissolved air de-trains and the flow depth of the two-phase mixture is reduced to that of single-phase flow. The section between the air entrainment, i.e. the entrance to the tailwater channel, to the point where the air has de-aerated completely, is termed the "de-aeration length". Apart from very small bubbles $(\mathrm{d}<0.1 \mathrm{~mm}$, micro-bubbles $)$, kept in suspension by turbulence and finally evacuated, no free undissolved air is present in the tailwater downstream of the de-aeration section.

Factors determining the rate of air entrainment are parameters such as the exit velocity at the Pelton bucket, the geometry of the turbine housing, the distance between the buckets and the tailwater level, etc. The air carried along into the tailwater needs replacing as otherwise the turbine runner would suck up an air/water mixture from below, which would involve a loss in turbine efficiency. Therefore, pipelines are provided to introduce air to the runner through openings in the turbine casing. This air has to be taken from the tailrace area, as the turbine housing and the tailrace channel form a closed system. Dissolved air in the water as a result of an increased ambient pressure is evacuated from the system. This air loss must continuously be compensated by the use of compressors.

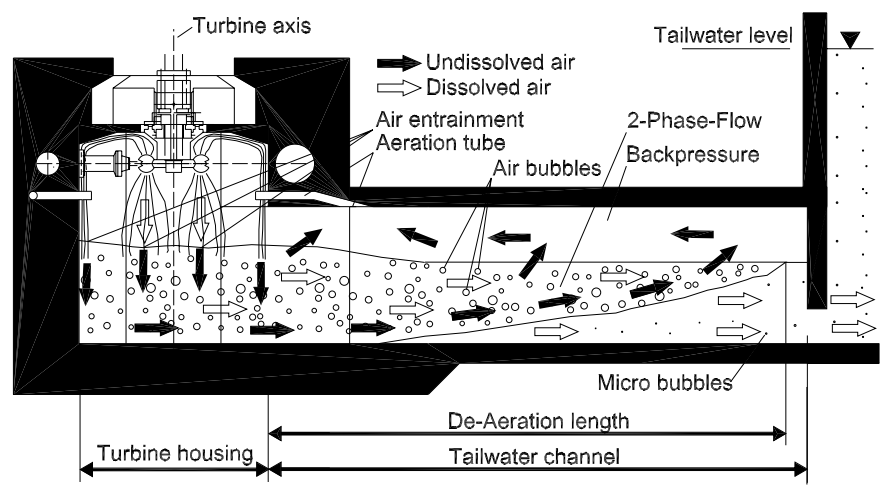

Figure 1: Schematic section through a vertical-shaft backpressure Pelton turbine.

Little is known at present about the influence of overpressure on air/water mixtures produced by a plunging free jet. For this reason, basic experiments have been conducted in a man-sized pressure chamber. The results will be summarised in the following paragraphs.

\section{Overview of the relevant literature}

Studies relating to the effects of an increase in ambient pressure on the processes of air entrainment and de-aeration in the turbine housing and the tailwater channel of Pelton turbines were conducted on a test rig by Ceravola and Noseda [1] in 1972. They built a turbine housing with runner and tailrace and operated it 
under different backpressures. The turbine discharges ranged from 66 to $100 \mathrm{l} / \mathrm{s}(\mathrm{H} \sim 120 \mathrm{~m})$. Figure 2 illustrates the flow conditions in the tailrace channel for different backpressures. The turbine housing, not shown, would be to the left of the photos. One can clearly see through the four windows that for a backpressure of $\mathrm{H}_{\mathrm{c}}=11.21 \mathrm{~m}$, corresponding to $2.1 \mathrm{bar}$ of absolute pressure, the white air/water mixture extends farther into the tailwater than for $\mathrm{H}_{\mathrm{c}}=0.54 \mathrm{~m}$.

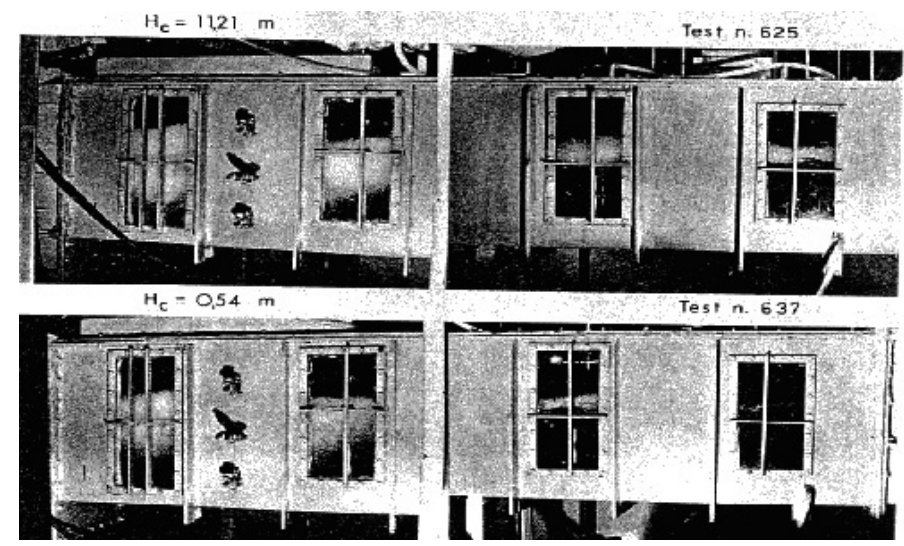

Figure 2: $\quad$ Model set-up by Ceravola / Noseda, tailrace, turbine discharge = approx. $88 \mathrm{l} / \mathrm{s}$, flow direction from left to right.

Based on this and comparable photo studies, the authors concluded that the propagation of the air/water mixture changed substantially as backpressure rose. The solution of air, and the increased air requirements in the turbine chamber involved, was considered to be the reason for the thorough mixing process and, hence, the increased air requirements. The information published did, however, not allow any quantitative conclusions.

Krishna [2] gave a summary of flow processes studied in bubble-column reactors. Air bubble swarms were made to rise vertically through the water fill at different pressure stages. The author found bubbles and bubble swarms to show different rise-velocity behaviours, depending on whether the bubbles were small $(\mathrm{d}<17 \mathrm{~mm})$ or large $(\mathrm{d}>17 \mathrm{~mm})$. Krishna concluded that the rise velocity of bubbles smaller than $17 \mathrm{~mm}$ were only weakly influenced by the greater air density (resulting from a higher pressure level).

Letzel et al. [3] demonstrated that an increased system pressure influenced the rise velocity of the larger bubbles in bubble column reactors. This is illustrated by the measurements of gas holdup $\varepsilon$ depending on superficial velocity $U$.

$$
\begin{gathered}
\varepsilon=\frac{\left(\mathrm{H}-\mathrm{H}_{0}\right)}{\mathrm{H}}[-] \\
\mathrm{U}=\frac{\mathrm{Q}_{\text {Air }}}{\mathrm{A}}[\mathrm{m} / \mathrm{s}]
\end{gathered}
$$

where $\mathrm{H}$ is the depth of the air/water mixture in the bubble-column reactor and $\mathrm{H}_{0}$ is the static water head of the static system, $\mathrm{Q}_{\text {Air }}$ is the air flow introduced at 
the bottom, and $\mathrm{A}$ is the cross-sectional area of the reactor. Figure 3 demonstrates that for rising system pressures, the increased gradient of the gas holdup $\varepsilon$ becomes steeper as the superficial velocity $U$ increases. This suggests that the rise velocity is lower in the case of larger bubbles and thus is a function of the bubble diameter. The lower rise velocity may be explained by the bubble diameter decreasing along with increasing pressure. In physical terms, a higher system pressure may be considered to reduce the stability of the larger bubbles. Based on the Kelvin/Helmholtz stability theory referring to the stability of a gas/liquid interface on the assumption of infinitesimal disturbances of the surface, and on the basis of experiments, Letzel et al. demonstrated that a correction factor DF had to be applied for calculating the rise velocity $\mathrm{V}_{\mathrm{b}}$ of large bubbles and gas holdup under pressures above atmospheric in order to allow for the changed air density $\rho_{\mathrm{G}}$. This is written as

$$
\mathrm{DF}=\frac{\mathrm{V}_{\mathrm{B}}}{\mathrm{V}_{\mathrm{B}, \mathrm{atm}}}=\frac{\varepsilon_{\mathrm{atm}}}{\varepsilon}=\sqrt{\frac{\rho_{\mathrm{atm}}}{\rho_{\mathrm{G}}}}[-]
$$

where $\mathrm{V}_{\mathrm{B}, \mathrm{atm}}$ is the bubble-rise velocity, $\varepsilon_{\mathrm{atm}}$ the gas holdup and $\rho_{\mathrm{atm}}$ the air density at atmospheric pressure.
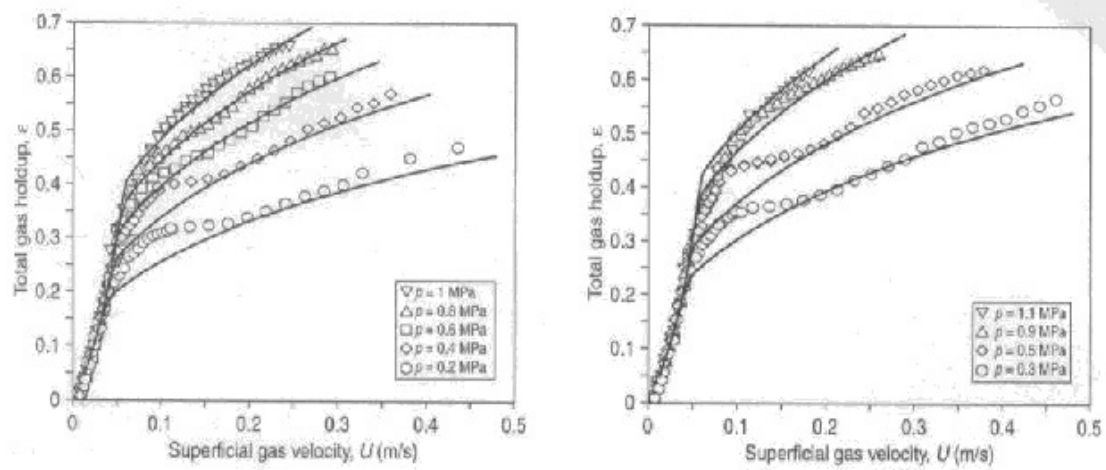

Figure 3: Influence of system pressure on gas holdup $\varepsilon$ (taken from [2]).

\section{Test set-up and tests}

\subsection{Test Set-up}

The test set-up was built in a man-sized pressure chamber. It consisted of a vertical test cuboid in plexiglass provided with a nozzle at the top, and a feeding pipe equipped with a ball valve, an outlet shaft installed at the bottom of the cuboid, a measuring channel with a calibrated triangular weir, a buffer tank, and a rotary pump with an $8 \mathrm{~m}$-long hose connection to the ball valve. The upright cuboid chamber, $374.4 \mathrm{~cm}^{2}$ in horizontal area and $116.5 \mathrm{~cm}$ high, was equipped with a measuring screen with a spacing of $5 \mathrm{~cm}$ installed at the front. A measuring tape was fixed to the side (Figure 4).

The rotary pump circulated the water at a discharge of up to $\mathrm{Q}_{\mathrm{Max}}=0.4 \mathrm{1} / \mathrm{s}$, depending on the position of the ball valve. A circular nozzle $5.25 \mathrm{~mm}$ in 
diameter produced a jet directed vertically downward. The exit velocity of the jet at the nozzle, calculated from the nozzle diameter and from the discharge measured at the triangular weir, was $19.3 \mathrm{~m} / \mathrm{s}$ at maximum system discharge. The maximum jet impact velocity was $19.6 \mathrm{~m} / \mathrm{s}$.
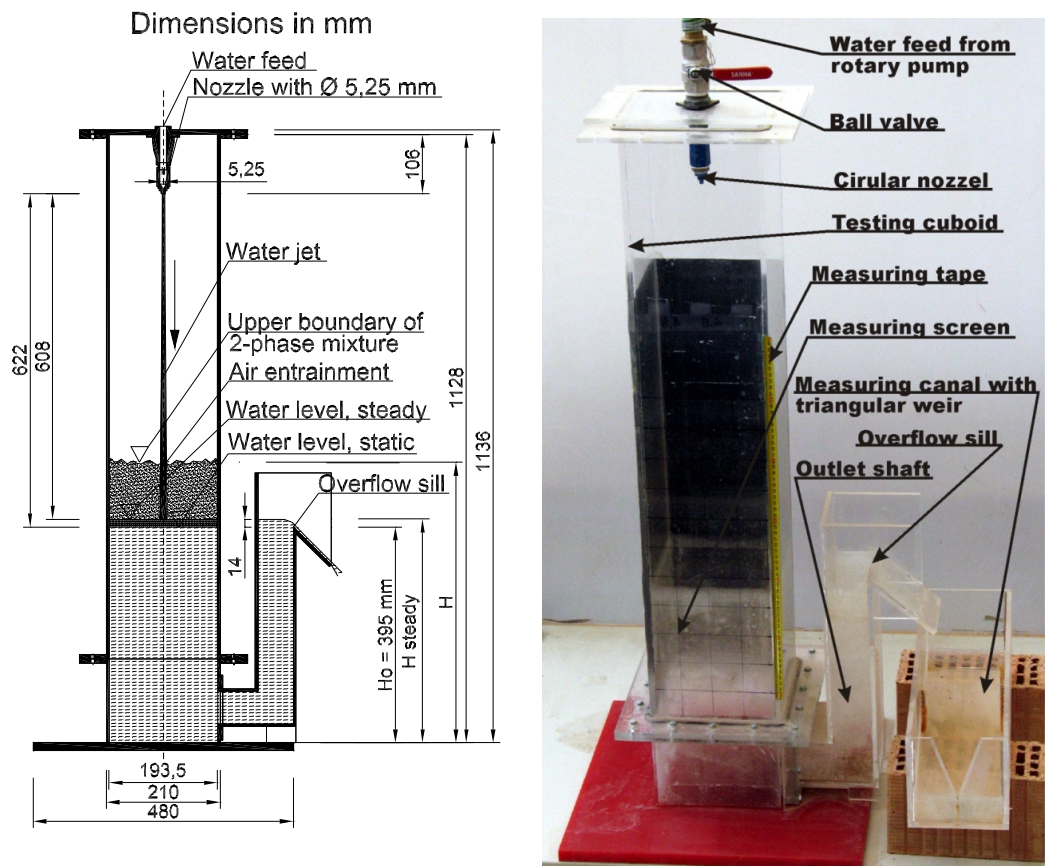

Figure 4: $\quad$ Test set-up.

At first, the cuboid was filled with water to a depth of $395 \mathrm{~mm}$, which was determined by the fixed overflow sill of the outlet shaft. The static water volume in the steady condition was 14.79 litres. The medium used was tap water having a temperature of $20^{\circ} \mathrm{C}$.

The steady water-volume condition within the cuboid was reached as a function of the respective discharge from the nozzle, dependent on the overflow characteristic of the overflow sill of the outlet shaft. After leaving the bottom of the chamber, the water climbed vertically through the outlet shaft and dropped over the overflow sill and into the measuring channel with its calibrated triangular weir (cone angle, $40^{\circ}$ ). The water flowing over the overflow sill was collected in a buffer tank of about 40-litre capacity. From there, the water was sucked in by the rotary pump and conveyed back to the nozzle through an $8 \mathrm{~m}$ long hose. The front of the chamber was filmed during the tests, using a video camera having a capability of 25 images per second.

\subsection{The tests}

Each test series, consisting of 13 tests, was run at constant absolute pressures of 1 bar (corresponding to atmospheric), 2.5 bar, and 4 bar, respectively, and at an 
ambient temperature of $20^{\circ} \mathrm{C}$ throughout. The pressures were raised from one stage to the next within about three minutes. During the pressure increases, an air condition system was used to disperse the compression heat generated in the process, so as to keep the temperature at a constant $20^{\circ} \mathrm{C}$.

In order to allow for the potential influence of air dissolving in water, the pump was shut off while the pressure was being raised in the chamber. During this process, the water surface was at rest. This was done to make sure that for each pressure stage, the first test was run with unsaturated water, since saturation is known to be greatly dependent on surface turbulence. Thus it was possible to draw conclusions with regard to the initial saturation and its influence on the behaviour of the air/water mixture.

During decompression from 4 bar to 1 bar, the pump kept working, and the depth of the two-phase mixture in the cuboid was measured for different pressures.

For each pressure level, 13 tests were run at different discharges, the first five tests with the rotary valve completely open, that is, at maximum discharge.

A test run consisted of (1) a phase of steady inflow from the nozzle for some 60 seconds and (2) the unsteady abrupt closing of the ball valve followed by a phase of some 15 seconds, during which the air bubbles raised to the surface and the two-phase mixture disintegrated. Analysis of the digital records allowed the determination of the rise velocity of the lower boundary of the bubble swarms, the gas holdup $\varepsilon$, the sinking velocity of the upper boundary of the air/water mixture, as well as the visual observation bubble size and the behaviour of the mixture.

\section{Test results}

\subsection{Steady conditions}

Figure 5 shows the air/water-mixture level under steady conditions and for a maximum system discharge prior to closing the rotary valve, for different ambient pressures.

The video analysis suggested that the water jet penetrating the mixture was slightly extended, the jet appeared to be torn apart to a greater width as the denser air passed through. The penetration depth of the jet was independent of the pressure. The limit ranged around 0.3 litres per second, or around a jetimpact velocity of $14.3 \mathrm{~m} / \mathrm{s}$ at the static water surface, for each pressure stage. This led to the conclusion that the influence of an increase in ambient pressure on jet turbulence could be ignored. It was seen that, as the pressure increased, the surface of the air/water mixture was higher and more unsteady and that the minimum and maximum air/water mixture levels took longer to reach the steady condition after opening the ball valve. The bubbles of the air/water mixture appeared to be larger around the transition zone between air and mixture. (Our test set-up did not permit exact determination of the bubble diameters).

Figure 6 is a graph showing gas holdup $\varepsilon$, plotted against the jet-impact velocities. In the case under study, with water being fed from above, the steady water level was used as the level $\mathrm{H}_{0}$ in formula (1) in order to account for the 
theoretically larger water volume resulting from water bulking under the overflow at the vertical shaft. (Note: As air bubbles were allowed to escape through the vertical shaft at the higher inflows, this should be considered when interpreting the $\varepsilon$ values.)

1 bar abs.

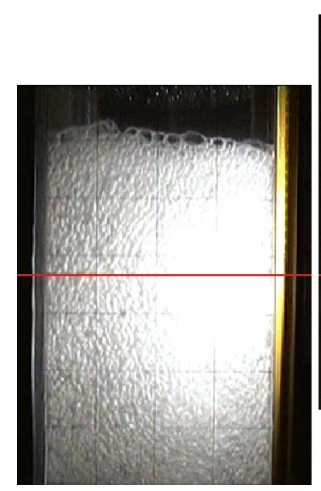

2.5 bar abs.

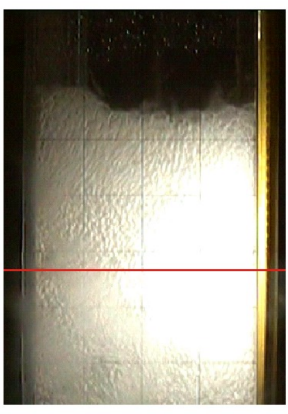

4 bar abs.

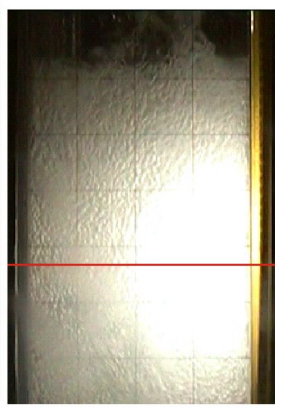

Figure 5: Steady condition, level of the air/water mixture for different pressures, continuous line indicates static water level.

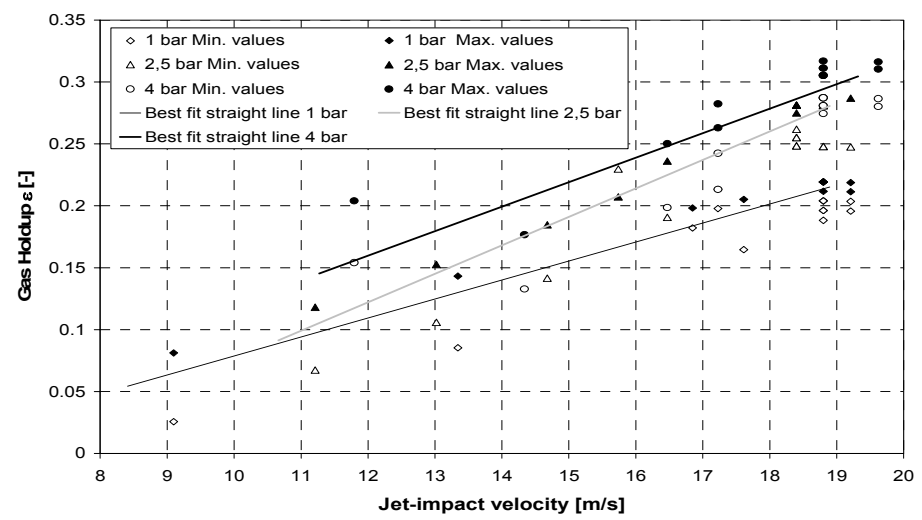

Figure 6: Gas holdup $\varepsilon$, plotted against jet-impact velocity at the steady water surface.

Minimum and maximum values are given in Figure 6 to account for the fact that the surface of the air/water mixture oscillated. In addition, best fit straight lines were entered into the diagram. As can be seen from the figure, gas holdup $\varepsilon$ increases along with a rising pressure. The first tests of each series gave no significantly higher or lower air/water mixture levels as compared with the tests that followed. Saturation can, therefore, be assumed to have no influence on hydrodynamic behaviour. Figure 7 shows gas holdup $\varepsilon$ plotted against pressure for maximum system discharge. Its value rose from about 0.21 for an absolute 
pressure of 1 bar to 0.30 for an absolute pressure of 4 bar. Compression is depicted by the continuous line, decompression is shown by the broken line. During the phase of rapid decompression from 4 bar to 2.5 bar, the formation of micro-bubbles increased as a result of air degassing from the water. The lines for $\varepsilon$ were practically identical for both decompression and compression.

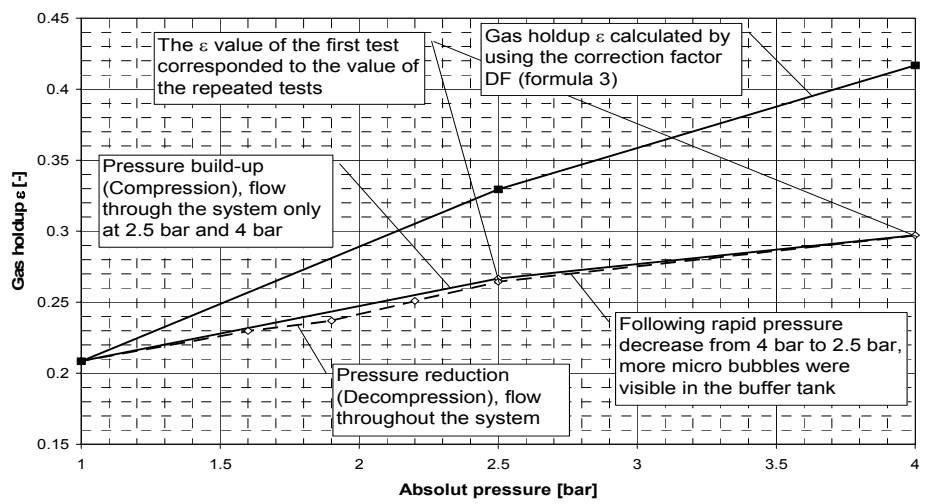

Figure 7: Gas holdup $\varepsilon$, for maximum system discharge, plotted against pressure.

Based on the measured value $\varepsilon$ for 1 bar and using the equation for the correction factor DF (formula 3), a theoretical gas holdup for the studied pressure levels has been calculated. Figure 7 shows the line for the calculated $\varepsilon$ values, where a deviation from the measured values with $24 \%$ for 2.5 bar and $40 \%$ for 4 bar was found. Comparing the measured and calculated values for the gas holdup $\varepsilon$ a general correlation could be achieved.

\subsection{Unsteady conditions}

Figure 8 is showing the upper and lower 2-phase boundary of the air/water mixture plotted against time, after system shut-down, for maximum discharge $\mathrm{Q}_{\text {Max }}=0.41 / \mathrm{s}$.

This graph demonstrates that for a raised ambient pressure, the upper boundary of the air/water mixture was about 1.4 and 1.62 times higher than at atmospheric pressure. However, the time needed for the air/water mixture to disintegrate remained at a constant 3 seconds, independently of the ambient pressure. The tests run at a low system discharge revealed, however, the ambient pressure to have a great impact on the disintegration time of the air/water mixture. The lower boundary of the air/water mixture, very much like the upper limit, was always higher under an increased ambient pressure than at atmospheric pressure.

The rise velocities of the bubble swarm, calculated from the slope of the lines representing the lower boundaries of the air/water mixture, were all situated within a range of about 10 to $12 \mathrm{~cm} / \mathrm{s}$ for all discharges and for each of the three pressure stages. This suggests that, for the ambient pressures used in the test, the 
rise velocity of the swarms of small bubbles is independent of the ambient pressure, while the test results showed the sinking velocity of the upper boundary of the air/water mixture to be a function of discharge and ambient pressure. That means that the sinking velocity rises along with an increase in backpressure and discharge (Figure 9), i.e. a faster disintegration of the 2-phase mixture. Sinking was seen to be initially fast under conditions of maximum discharge. By the time the entrained air had de-aerated completely, the sinking velocity had decreased by about $50 \%$. Further studies have shown the upper air/water mixture boundary to sink fairly evenly under conditions of low discharge (Figure 9).

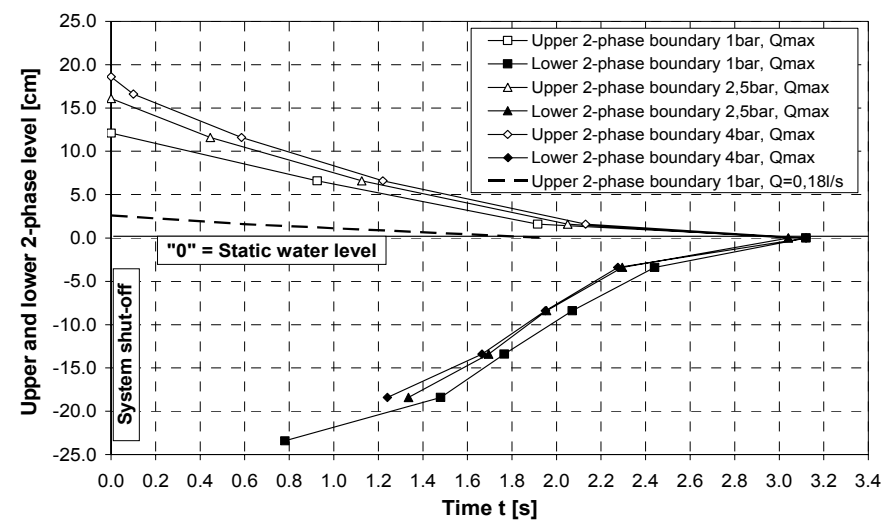

Figure 8: Mean decrease of the upper and lower boundaries of the air/water mixture for maximum system discharge $Q=0.41 / \mathrm{s}$ (extract from all the test measurements).

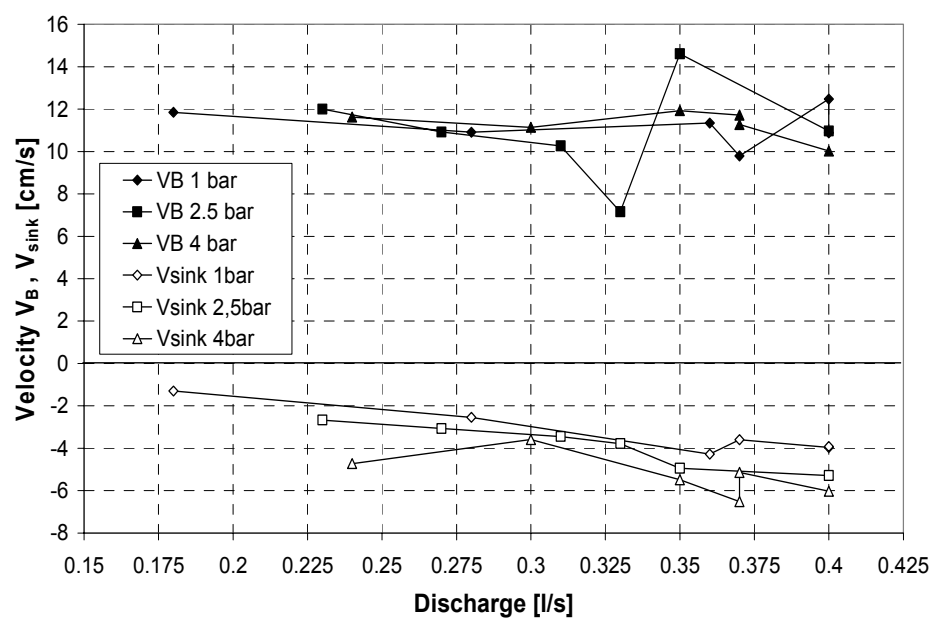

Figure 9: $\quad$ Rise velocity $V_{B}$ of the bubble swarm and mean sinking velocity $\mathrm{V}_{\text {sink }}$ of the upper air/water mixture boundary for ambient pressures of 1 bar, 2.5 bar and 4 bar. 


\section{Summary and conclusion}

Basic tests were conducted in a pressure chamber to study the effects of an increase in air pressure on the air/water mixture in the tailwater below Pelton turbines operating in backpressure mode. The results showed a rise in ambient pressure to increase the volume of the air/water mixture generated by a water jet. Gas holdup $\varepsilon$ was seen to increase by a maximum of $55 \%$. For a plant equipped with Pelton turbines operating under backpressure, this may mean that the clearance between the upper boundary of the air/water mixture in the tailrace channel and the runner decreases as the backpressure increases. This would reduce the safety margin allowed for in the design of the turbine clearance. Where the selected clearance is too small, the runner risks sucking the air/water mixture in. The performed tests have supplied no direct quantitative results regarding the increase of the air/water mixture below the Pelton turbine. No difference was found to exist for $\varepsilon$ between the initial tests and the remaining tests within each series, representing the conditions of initial saturation and complete saturation. This allows the conclusion that in pumped-storage plants operated under conditions of hydraulic short-circuit, the flow depths of the air/water mixture under backpressure conditions, at maximum discharge, will form independently of the degree of saturation, that is, independently of the load condition.

Analysis of the de-aeration process of the air/water mixture revealed the mean rise velocities of the bubble swarm to range between $10 \mathrm{~cm} / \mathrm{s}$ and $12 \mathrm{~cm} / \mathrm{s}$, independently of the ambient pressure. The time needed for the undissolved air components to de-train and, hence, the de-aeration length, is, therefore, not expected to increase even under changed backpressure conditions. The design and location of the turbine aerators as well as the channel geometry from which the air has to be taken should allow for the increased level of the air/water mixture in the tailwater, as otherwise an air/water mixture might be sucked in instead of pure air.

\section{References}

[1] O. Ceravola and G. Noseda, Back pressure operation of Pelton turbines for ternary units in pumped-storage plants, IAHR-Symposium Rome, paper C3, 1972 (including discussion).

[2] R. Krishna, A scale-up strategy for a commercial scale bubble column slurry Reactor for Fischer-Tropsch Synthesis, Oil \& Gas Science and Technology, Vol. 55 (2000), No. 4, pp. 359-393.

[3] Letzel et al., Influence of elevated Pressure on the Stability of Bubble Flows, Chem. Eng. Sci., Vol. 52, pp.3733-3739. 